\title{
Bilateral Fusion of Deciduous Teeth in Mandibular Arch with the Absence of Permanent Successor: Concomitant Fusio-agenesia
}

\author{
Pratik B Kariya ${ }^{1}$, Swara Shah ${ }^{2}$, Adrija Buch ${ }^{3}$, Romil B Shah ${ }^{4}$
}

\begin{abstract}
Tooth fusion is a common type of a developmental dental anomaly that can be described as the union of two embryologically developing teeth at varying stages to its development. It is commonly seen in deciduous dentition, more frequently unilaterally, and in the anterior region. Various terms such as connate teeth, double formations, synodontia, or conjoined teeth are often used to describe tooth fusion. The prevalence of fusion in deciduous teeth is reported to be around $0.5-2.5 \%$ and only $0.02 \%$ for bilateral involvement of deciduous teeth. Early diagnosis of such conditions is important as it may cause various clinical problems, such as malocclusion, esthetic concerns, periodontal conditions, and dental caries. This paper reports a rare case of concomitant occurrence of bilaterally fused deciduous mandibular lateral incisor and canine with congenital agenesis of permanent mandibular lateral incisors bilaterally.

Keywords: Concomitant, Congenitally missing teeth, Deciduous teeth, Fusion, Tooth agenesis.

Journal of South Asian Association of Pediatric Dentistry (2020): 10.5005/jp-journals-10077-3046
\end{abstract}

\section{INTRODUCTION}

Teeth have complex anatomic structures that may encounter various anomalies in its due course of development, such as defects in their shape, size, structure, and number. Tooth fusion is one of the most unusual forms of anomalies involving shape. ${ }^{1}$

In 1963, Tannenbaum and Ailing defined tooth fusion as a union of two separate tooth buds at some stage in their development. ${ }^{2}$ Fusion is commonly recognized as the unification of two discrete dental buds, and the condition may arise at any stage during the developmental process of the dental organ. ${ }^{3}$ The effect of pressure or physical forces producing close proximity between two developing tooth buds has been speculated as one of the predictable causes for fusion. ${ }^{4}$ Clinically, teeth may appear as single normal or large-sized teeth, depending upon the stage at which embryological union occurs during the development.

Tooth fusion is commonly misdiagnosed with gemination, later being a complete division of one tooth bud into two. ${ }^{5}$ Tooth fusion may be seen in both deciduous and permanent dentitions, though tooth fusion is a more prevalent finding in deciduous dentition. ${ }^{6}$ Various studies from the USA and Japan have shown that the prevalence rate of tooth fusion is $0.5-2.5 \%$, respectively, as compared to $0.1 \%$ in permanent dentition. Tooth fusion may be unilateral or bilateral. Bilateral tooth fusion occurrence is even more rare, with $0.02 \%$ prevalence rate in deciduous dentition. ${ }^{7}$ There is no sex predilection reported in the case of fusion. However, mandibular incisors are common sites of occurrence. Genetics may have a role in the increased incidence of tooth fusion. Fusion may affect two normal teeth or it may also occur between a normal tooth and a supernumerary tooth. ${ }^{8}$

The presence of fused teeth can cause numerous clinical problems like unacceptable appearance, periodontal conditions, malocclusion, or dental caries. A frequent finding associated with fusion of primary teeth is the congenital absence of its successor permanent teeth. Unilateral fusion of the lateral incisor and cuspid is common in the deciduous dentition, ${ }^{9}$ but bilateral dental fusion in
${ }^{1-3}$ Department of Paedodontics and Preventive Dentistry, KM Shah Dental College and Hospital, Sumandeep Vidyapeeth, Vadodara, Gujarat, India

${ }^{4}$ Department of Orthodontics and Dentofacial Orthopedics, KM Shah Dental College, Vadodara, Gujarat, India

Corresponding Author: Pratik B Kariya, Department of Paedodontics and Preventive Dentistry, KM Shah Dental College and Hospital, Sumandeep Vidyapeeth, Vadodara, Gujarat, India, Phone: +91 9558330199, e-mail: prateek.kariya@gmail.com

How to cite this article: Kariya PB, Shah S, Buch S, et al. Bilateral Fusion of Deciduous Teeth in Mandibular Arch with the Absence of Permanent Successor: Concomitant Fusio-agenesia. J South Asian Assoc Pediatr Dent 2020;3(2):80-83.

Source of support: Nil

Conflict of interest: None

the deciduous dentition is a rare dental anomaly. ${ }^{10}$ This case report presents a concomitant occurrence of bilaterally fused deciduous mandibular lateral incisor and canine with bilateral congenital agenesis of permanent lateral incisors.

\section{Case Description}

A six-year-old boy reported with a chief complaint of unusually large-sized teeth in the lower front tooth region (Fig. 1). Intraoral examination revealed the presence of unusually large teeth in the mandibular anterior region bilaterally. Deep labiolingual grooves were associated with both the enlarged teeth (Fig. 1). These deep grooves on the labial and lingual surfaces were not affected either by dental caries or by periodontal problems. Physiologic space was also present between mandibular central incisors and fused lateral incisor and canine on the both sides. A total number of teeth in mandibular arch were eight and the parents did not give any history of early exfoliation of any deciduous teeth, which was 


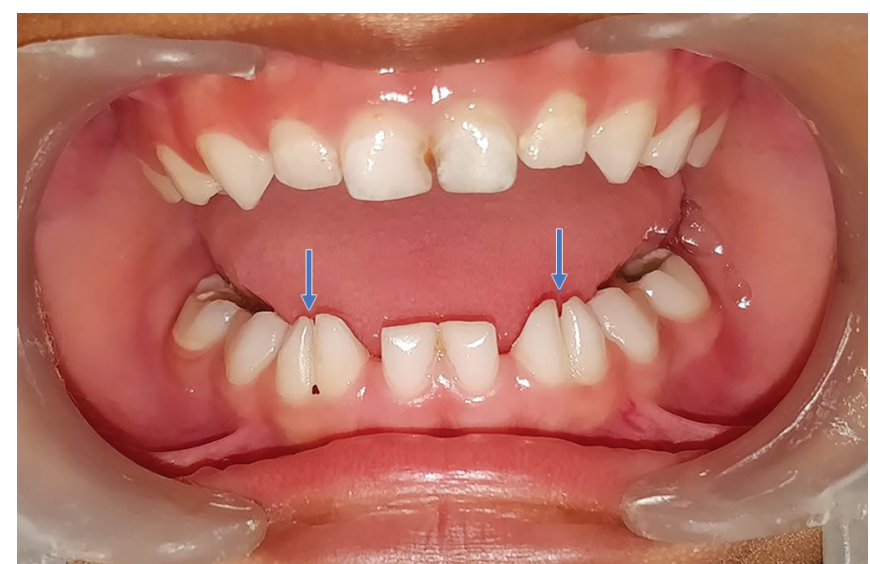

Fig. 1: Intraoral photograph showing bilaterally fused mandibular deciduous lateral incisor and canine

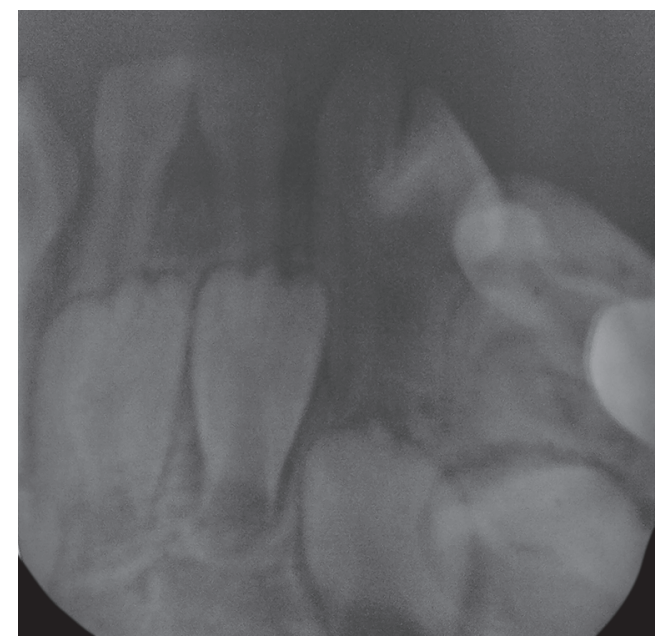

Fig. 3: Intraoral periapical radiograph showing fusion between mandibular left deciduous lateral incisor and canine

strongly suggestive of fused deciduous lateral incisor and canine (72-73 and 82-83). No relevant medical history was found with his condition.

For radiographic evaluation, both intraoral periapical (IOPA) radiograph and orthopantomogram (OPG) were taken. Intraoral periapical radiograph revealed bilaterally fused deciduous mandibular lateral incisor and canine (72-73 and 82-83) with an incomplete fusion of two crowns and completely fused roots with two separate root canals, thereby indicating that the fusion was of incomplete type (Figs 2 and 3). The OPG showed bilaterally fused deciduous mandibular lateral incisor and canine (72-73 and 82-83) and congenitally missing permanent lateral incisor bilaterally (Fig. 4). Multisurface caries were seen in relation to mandibular second primary molars of left and right sides (75 and 85 ).

Instructions regarding the maintenance of oral hygiene were given to the patient to avoid caries. A multidisciplinary approach that included orthodontic treatment to ensure functional occlusion and esthetics was explained to the parents. After parental consent, oral prophylaxis was done followed by the restoration of mandibular second primary molars of left and right sides (75 and 85 ) with stainless steel crowns (Size 4, 3M, ESPE, USA) due to

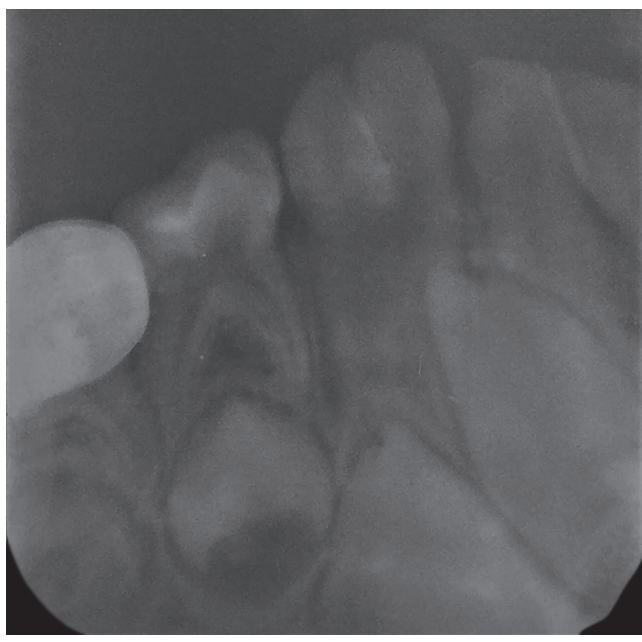

Fig. 2: Intraoral periapical radiograph showing fusion between mandibular right deciduous lateral incisor and canine

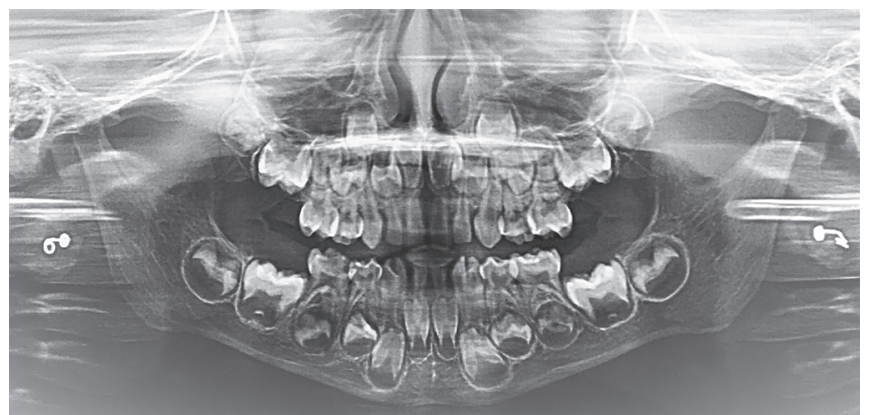

Fig. 4: Orthopantomogram showing bilaterally fused mandibular deciduous lateral incisor and canine and congenitally missing permanent lateral incisor

the presence of multisurface caries. Subsequently, fissure sealants (Clinpro ${ }^{\circledast}$ M St Paul, MN) with respect to maxillary second primary molars of left and right sides (55 and 65) and topical fluoride application (1.23\% APF Gel, Sultan Healthcare Inc., USA) were done, with every six-month periodic follow-up (Fig. 5).

\section{Discussion}

Tooth fusion refers to the union of two separate tooth germs in its due course of the development. Tooth fusion may be complete or incomplete, depending on the stages of tooth development and time of union. Fusion can be seen in either deciduous dentition or permanent dentition. Fusion may be seen involving two normal teeth as in the present case or sometimes between a normal tooth and supernumerary tooth. ${ }^{8}$

Tooth fusion can be classified into two types-complete and incomplete. Complete fusion begins before calcification of tooth germ - the crown incorporates features of both participating teeth with regard to their enamel, dentin, cementum, and pulp, whereas incomplete fusion occurs at a later stage of tooth calcification-the tooth might exhibit separate crowns and fusion may be limited to the roots alone with pulp canals fused or separate. ${ }^{1}$

Tooth fusion poses numerous related problems like esthetics and malocclusion, possible loss of arch length, delayed or ectopic 


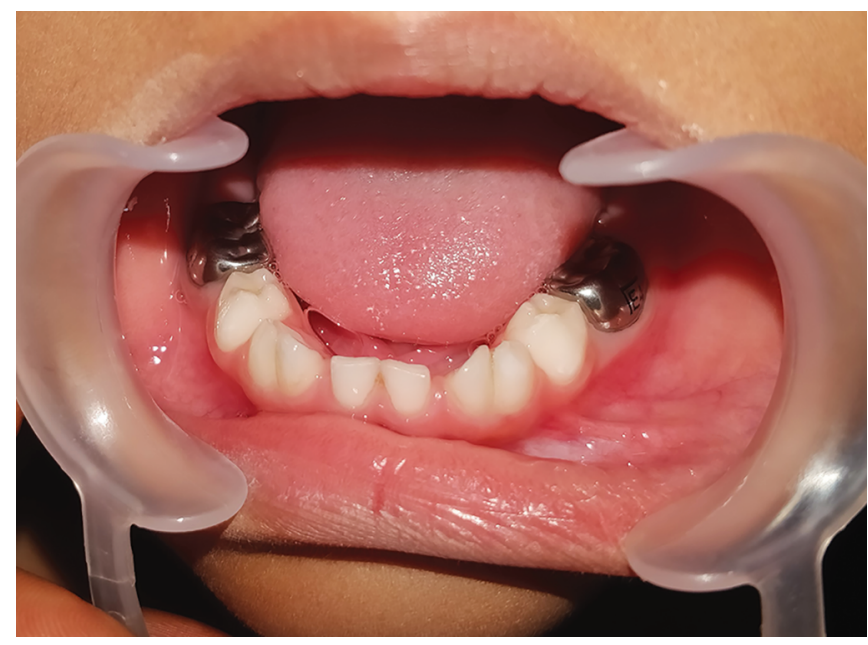

Fig. 5: Postoperative intraoral photograph showing mandibular second primary molars of left and right sides (75 and 85 ) treated with stainless steel crowns

eruption of the permanent successor teeth, caries along the line of demarcation, early pulp exposure due to deep grooves, and periodontal abscesses. Greater surface area of fused primary teeth may delay or alter its resorption rate or direction which in turn may lead to deflection in eruption or impaction of permanent successor. Strict oral hygiene is imperative to maintain periodontal health. ${ }^{5}$

Very few cases of fusion in mandibular deciduous dentition have been reported in the Indian population, ${ }^{1,11}$ though cases of bilateral fusion are less frequent. Tewari and Pandey reported bilateral fusion of mandibular deciduous central and lateral incisors with the absence of bilateral permanent central incisors, ${ }^{12}$ whereas Chachra and Sharma reported the absence of both permanent mandibular lateral incisors with the bilateral fusion of mandibular primary central and lateral incisors and ${ }^{13}$ Acikel and Hagman reported that cases with fusion in deciduous dentition have a $75 \%$ chance of lacking the succedaneous lateral incisor. ${ }^{14,15}$ The same is observed in our case also where permanent lateral incisors are bilaterally missing. However, few studies reported no alteration in the development of permanent successors when the fusion is involved in the primary mandibular central and lateral incisors. ${ }^{16,17}$

Various etiological factors like physical forces or pressure causing the close proximity of developing teeth, trauma, and viral infection during the pregnancy, environmental conditions, or genetic basis possibly autosomal dominant with reduced penetrance could be attributed to tooth fusion. Fusion has also been reported along with congenital anomalies such as cleft lip and with X-linked congenital conditions. ${ }^{14}$

Humans have two sets of dentitions, i.e., diphyodont. The first being primary dentition which is considered as predecessor and the next set is of permanent dentition which is considered as successors. These successional teeth are developed from the extension of the dental lamina of their predecessor tooth in its early cap stage by activating SOX $2+$ cells. Agenesis of successional teeth may result due to failure in the initiation of tooth formation, decreased odontogenic potential of the dental lamina, or arrested development during the early stage. The possibility in the case of missing successional teeth following fused primary teeth (concomitant fusio-agenesia) can be two fused teeth together act as a single unit that might signal the initiation of the only single successional tooth. Wnt/ $\beta$-catenin signaling regulates tooth development at multiple stages and has been suggested as the key regulator of successional tooth formation. The inhibition of Wnt/ $\beta$-catenin signaling in the early epithelium leads to arrested tooth development at the lamina stage. Fusion of primary teeth could be one of the reasons behind the inhibition of these signals leading to concomitant fusio-agenesia. ${ }^{18}$

The treatment of fused teeth depends on the patient's orthodontic, periodontal, esthetic, and functional requirements. Types of teeth involved, type of fusion, i.e., complete or incomplete, and its morphologic results govern the management of a case. If the fused tooth is free from caries, as in the present case, it may not require any particular treatment and only universal preventive advice should be given to the parent and the child. In the case of the presence of caries, restoration should be placed for the retention of its function and esthetics. In the cases where there are esthetic concerns with the absence of other underlying risk factors, conservative use of tooth reshaping, direct composite bonding, bonded porcelain veneers, and crowns have been described. ${ }^{19}$ Surgical intervention, such as extraction of fused teeth, could be the treatment of choice in the cases where there are high risks of malocclusion development. ${ }^{20}$ In our case, parents were made aware of a multidisciplinary approach that included orthodontic treatment to ensure functional occlusion and esthetics if required. These conditions require a minimal intervention approach, preventive procedures, surgical management, and long-term follow-up. The clinical observation, early diagnosis with orthopantomography, periapical radiographs with prompt intervention, and long-term follow-up significantly improve the prognosis and treatment outcomes.

\section{Conclusion}

Careful monitoring of the condition is recommended to avoid a potential malocclusion in permanent dentition and to maintain these teeth sound and caries-free until the eruption of the permanent dentition. Genetic factor that fused teeth together act as a single unit that signals the initiation of a single successional tooth can be attributed as a possible etiology of missing successional teeth following fused primary teeth, concomitant fusio-agenesia. Successful management of such cases depends on the early diagnosis, morphology of fused teeth, knowledge and skills of the practitioner, and prompt intervention.

We propose the term "concomitant fusio-agenesia" for condition showing fusion in deciduous dentition along with its congenitally missing permanent successor.

\section{How this Paper is Important for Dental FRATERNITY}

- A thorough clinical and radiographic evaluation is compulsory as early diagnosis results in enhanced prognosis.

- Early diagnosis and prompt intervention of fused teeth is essential part of preventive dentistry as it can avoid various related complications periodontal problems, malalignment, impaction of permanent successor, etc. 


\section{References}

1. Pereira T. Fusion of a primary mandibular lateral incisor and canine: A rarity. Arch Med Health Sci 2017;5(2):258-260. DOI: 10.4103/amhs. amhs_78_17.

2. Tannenbaum KA, Ailing EE. Anomalous tooth development: case reports of gemination and twinning. Oral Surg Oral Med Oral Pathol 1963;16(7):883-887. DOI: 10.1016/0030-4220(63)90326-8.

3. Neena IE, Sharma R, Poornima $P$, et al. Gemination in primary central incisor. J Oral Res Rev 2015;7(2):55-57. DOI: 10.4103/2249-4987.172495.

4. Rajashekhara BS, Dave B, Manjunatha BS, et al. Bilateral fusion of primary mandibular lateral incisors and canines: $A$ report of a rare case. Rev Odontol Cienc 2010;25(4):427-429. DOI: 10.1590/S198065232010000400020.

5. Gupta T, Manuja N. Bilateral fusion of primary mandibular incisors: A rare case report. J Clin Diagn Res 2015;9(12):ZJ01. DOI: 10.7860/ JCDR/2015/16077.6933.

6. Neville BW, Daman DD, Allen CM, et al. Oral and Maxillofacial Pathology. 2nd ed., Philadelphia: W.B. Saunders; 2002. pp. 74-77.

7. Duncan WK, Helpin ML. Bilateral fusion and gemination: a literature analysis and case report. Oral Surg Oral Med Oral Pathol 1987;64(1):82-87. DOI: 10.1016/0030-4220(87)90121-6.

8. Khan R, Kariya PB, Mallikarjuna R, et al. Fusion of permanent maxillary right central incisor and mesiodens in an 8-year-old child. BMJ Case Rep 2015. DOI: 10.1136/bcr-2014-208541.

9. Eidelman E. Fusion of maxillary primary central and lateral incisors bilaterally. Pedia Dent 1981;3(4):346-347.

10. Joshi V, Pavankumar K, Ramana V, et al. Bilateral fusion of the mandibular primary incisors: A case report. Int J Oral Max Patho 2011;2(2):40-43.
11. Mohtesham I, Shakil M, Jose $M$, et al. Fusion of deciduous central incisors. Arch Med Health Sci 2015;3(1):85-87. DOI: 10.4103/23214848.154951.

12. Tewari N, Pandey RK. Bilateral fusion in primary mandibular teeth: a report of two cases. J Indian Soc Pedod Prev Dent 2011;29(1):50-52. DOI: 10.4103/0970-4388.79936.

13. Chachra S, Sharma AK. Bilateral fusion of the mandibular primary incisors: A case report. J Indian Dent Assoc 2011;5:248-249.

14. Acikel $H$, Ibis $S$, Tunc ES. Primary fused teeth and findings in permanent dentition. Med Princ Pract 2018;27(2):129-132. DOI: 10.1159/000487322.

15. Hagman FT. Anomalies of form and number, fused primary teeth, a correlation of the dentitions. ASDC J Dent Child 1988;55(5):359-361.

16. Wu CW, Lin YT, Lin YT. Double primary teeth in children under 17 years old and their correlation with permanent successors. Chang Gung Med J 2010;33(2):188-192.

17. Nik-Hussein NN, Abdul Majid Z. Dental anomalies in the primary dentition: distribution and correlation with the permanent dentition. J Clin Pediatr Dent 1996;21(1):15-19.

18. Juuri $E$, Balic $A$. The biology underlying abnormalities of tooth number in humans. J Dent Res 2017;96(11):1248-1256. DOI: 10.1177/0022034517720158.

19. Eregowda N, Singh S, Poornima P, et al. Mandibular unilateral fusion in primary dentition. J Oral Res Rev 2017;9(1):29-31. DOI: 10.4103/jorr. jorr_35_16.

20. Bernardi S, Bianchi S, Bernardi G, et.al. Clinical management of fusion in primary mandibular incisors: a systematic literature review. Acta Odontol Scand 2020. 1-8. DOI: 10.1080/00016357.2020. 1734233. 